\title{
Beam spread functions calculated using Feynman path integrals
}

\author{
Paul Kilgo, Jerry Tessendorf \\ School of Computing \\ Clemson University \\ 100 McAdams Hall, Clemson, S.C. 29634 \\ pkilgo@clemson.edu; jtessen@clemson.edu
}

\begin{abstract}
A method of solving the radiative transfer equation using Feynman path integrals (FPIs) is discussed. The FPI approach is a mathematical framework for computing multiple scattering in participating media. Its numerical behavior is not well known, and techniques are being developed to solve the FPI approach numerically. A missing numerical technique is detailed and used to calculate beam spread functions (BSFs), a commonly studied experimental property of many types of media. The calculations are compared against measured BSFs of sea ice. Analysis shows differently-shaped BSFs, and suggests the width parameter of the calculated BSF's Gaussian fit approaches a value in the limit of the number of path segments. A projection is attempted, but suggests a larger number of path segments would not increase the width of the calculated BSF. The trial suggests the approach is numerically stable, but requires further testing to ensure scientific accuracy.
\end{abstract}

Keywords: path integral, radiative transfer, participating media, beam spread function

\section{Introduction}

Multiple scattering is sometimes expressed as a large collections of paths through participating media. Because there are very few restrictions on paths radiation can take through the media, the total path space to explore is quite large. Feynman path integrals (FPI) applied to radiative transfer $[1,2,3,4]$ is a mathematical model for radiation transport through participating media which is general and suitable for many applications. For instance, Perelman et al. $[5,6,7,8]$ have modeled light transport using path integrals with intended application in optical tomography, though much of their work uses the path integral model as theory to compare with experimental or Monte Carlo results rather than directly evaluating the path integral. The key difficulties in directly evaluating the path integral in our approach are the lack of known numerical methods for solving it and immense amount of computer time which is needed to arrive at a solution. Recent work [9] had revisited and sped up a numerical method used for perturbing paths and analyzed its statistical behavior, proving repeatability and unbiased Monte Carlo calculation. Numerical methods for creating initial paths to use as inputs for the perturbation algorithm were not yet defined, which is a necessary step for more complex experiments, for example, to validate the technique against experimental data.

Beam spread functions (BSFs) and point spread functions (PSFs) are equivalent [10], wellstudied optical properties of scattering media such as sea ice [11] and ocean water [12]. Mertens and Replogle [13] give thorough definitions of both and their application to optics. They are geometrically simple and typically studied in cases which can be approximated by a uniformly scattering and infinite media. Multiple scattering effects are apparent in the higher-angle portions of a BSF or PSF plot, manifesting as a gradual decrease in the 
intensity from a single peak at $0^{\circ}$. This makes the BSF and PSF attractive options for comparison of computational radiative transfer solutions.

To summarize the scope of work completed in the measurement of both BSFs and PSFs, each has been measured for various types of media in both laboratory and field settings. This has yielded some results which computational techniques can use for validation. Some of the earliest work was by Duntley [12] with laboratory PSF measurements in highly scattering water tanks. Voss [14] measured the PSF of the Sargasso Sea water. Schoonmaker et al. [15] measured the BSF of laboratory-grown sea ice, and were able to fit a Gaussian model to their data. Maffione et al. [11] measured the BSF of Alaskan sea ice and attempted a Gaussian fit but found a Lorentzian form function which fit their data.

Because of the available data on BSF measurement, much of this article focuses on the comparison of the FPI approach to measured sea ice BSFs, particularly those measured by Maffione et al. We use this data because of the many different depth levels and radii at which it is measured, and the provided models for Gaussian and Lorentzian functions to describe the data. By using the same models, we can begin to make comparisons with the experimental data and determine if the FPI approach is capable of modeling physical phenomena.

The rest of this article is as follows. Section 2 provides background on the FPI approach and its mathematical foundations. Section 3 presents a recently developed numerical technique to create "seed paths" for integration over some of the spatial integrals in the FPI approach. Section 4 presents a comparison the BSFs calculated by the FPI approach to those measured by Maffione et al. Finally, Section 5 presents concluding remarks and future directions.

\section{Path integrals for radiative transfer}

Using Feynman path integrals for solving radiative transfer was first introduced by Tessendorf in 1987 [1] who also studied its numerical behavior in 2009 [3]. This report gives a derivation of the mathematical model of volume rendering which is derived from the radiative transfer equation,

$$
\begin{aligned}
L\left(\vec{x}_{1}, \hat{n}_{1}\right)= & \int_{0}^{\infty} d s \int d^{3} \vec{x}_{0} \int_{4 \pi} d \Omega_{0} \\
& \times G\left(s, \vec{x}_{1}, \hat{n}_{1}, \vec{x}_{0}, \hat{n}_{0}\right) S\left(\vec{x}_{0}, \hat{n}_{0}\right),
\end{aligned}
$$

where $G$ is the transport kernel and $S$ is a source function. The transport kernel gives a proportion of the radiation transported from the source function at position $\vec{x}_{0}$ and direction $\hat{n}_{0}$ traveling a over an arbitrary time variable $s$ and ending at a position $\vec{x}_{1}$ and direction $\hat{n}_{1}$. In this article we refer to the parameters of the transport function as the boundary constraints as they are fixed when calculating the path integral which defines $G$. The source function gives the physical quantity of light emitted from position $\vec{x}_{0}$ and direction $\hat{n}_{0}$. For the purposes of the FPI approach, we will assume $s$ is the arc length of a photon of interest's flight path. This equation for the radiance at $\vec{x}_{1}$ in direction $\hat{n}_{1}$ tells us we must integrate $G$ over all space, over all directions, and over all arc lengths to compute an answer.

Even if the boundary constraints are fixed, there are several possible paths that satisfy these constraints. Therefore, calculating $G$, the transport kernel, is still a matter to discuss. The FPI approach says the transport kernel has a representation in the form of a Feynman path integral. We can define a path as a space curve $\mathbf{x}\left(s^{\prime}\right)$, $0 \leq s^{\prime} \leq s$, having unit tangent vectors

$$
\hat{\beta}\left(s^{\prime}\right)=\frac{d \mathbf{x}\left(s^{\prime}\right)}{d s^{\prime}} .
$$

The path integral portion only will accept space curves satisfying the boundary constraints set by the outermost integrals in Equation (1). Therefore, the constraints for the space curve are

$$
\begin{gathered}
\hat{\beta}(0)=\hat{n}_{0} \\
\hat{\beta}(s)=\hat{n}_{1} \\
\vec{x}_{1}-\vec{x}_{0}=\int_{0}^{s} d s^{\prime} \hat{\beta}\left(s^{\prime}\right) .
\end{gathered}
$$

We also define scalar field functions to represent the optical properties of the media. They are 
$a(\vec{x})$, the absorption coefficient; $b(\vec{x})$, the scattering coefficient; and $c(\vec{x})=a(\vec{x})+b(\vec{x})$, the total extinction. Though, in this article we consider the case where these are not spatially varying. We simplify the notation by writing $a, b$, and $c$ respectively in this case. The path integral form of $G[3]$ is

$$
\begin{aligned}
G\left(s, \vec{x}_{1}, \hat{n}_{1}, \vec{x}_{0}, \hat{n}_{0}\right)= \\
\quad \int[d \Omega][d \mathbf{p}] \\
\quad \times \delta\left(\hat{\beta}(0)-\hat{n}_{0}\right) \delta\left(\hat{\beta}(s)-\hat{n}_{1}\right) \\
\quad \times \delta\left(\vec{x}_{1}-\vec{x}_{0}-\int_{0}^{s} d s^{\prime} \hat{\beta}\left(s^{\prime}\right)\right) \\
\quad \times \exp \left(-\int_{0}^{s} d s^{\prime} c\left(\mathbf{x}\left(s^{\prime}\right)\right)\right) \\
\quad \times \exp \left(i \int_{0}^{s} d s^{\prime} \mathbf{p}\left(s^{\prime}\right) \cdot \frac{d \hat{\beta}\left(s^{\prime}\right)}{d s^{\prime}}\right) \\
\quad \times \exp \left(\int_{0}^{s} d s^{\prime} b\left(\mathbf{x}\left(s^{\prime}\right)\right) \tilde{Z}\left(\left|\mathbf{p}\left(s^{\prime}\right)\right|\right)\right) .
\end{aligned}
$$

We adopt the notation $[d \Omega]$ and $[d \mathbf{p}]$ to represent an integration over all the $\hat{\beta}(s)$ and $\mathbf{p}(s)$ functions; the delta functions force satisfaction of Equations (3) to (5). The term $\tilde{Z}$ is the phase function of the media, transformed into a Fourierlike form and $\mathbf{p}$ is also introduced from this transformation. This article uses a forward-peaked Gaussian phase function for $\tilde{Z}$ because of its exact analytic solution and computational ease, but the general phase function is acceptable for use in the FPI approach. A Gaussian phase function is not strictly accurate for scientific application, but at this point we are trying to show computational feasibility for the FPI approach in a plausible test scenario.

Earlier work [9] had focused on the statistical behavior of the path integral in the transport kernel itself, but offered no methods for constructing space curves which meet the constraints posed by the delta functions. In this work we explore the behavior as we integrate over the path integral itself as well as the spatial and solid angle distributions of a BSF. We introduce new numerical methods to accomplish this. Using the new techniques we can begin to compare against experimentally acquired BSFs.

\section{Numerical Methods}

We develop a strategy for generating space curves matching constraints posed by the path integral. To calculate the BSF of spatially uniform media, we can set $\vec{x}_{0}$ and $\hat{n}_{0}$ to arbitrary constants. We use $\vec{x}_{0}=\overrightarrow{0}$ and $\hat{n}_{0}=\hat{x}$. We choose $\vec{x}_{1}$ by placing a sphere of radius $R$ centered at $\vec{x}_{0}$ and selecting $\vec{x}_{1}$ and $\hat{n}_{1}$ using spherically uniform sampling,

$$
\begin{gathered}
\hat{n}_{1}=\hat{x} \eta_{1}+\hat{y} \cos \left(2 \pi \xi_{1}\right) \sqrt{1-\eta_{1}^{2}} \\
+\hat{z} \sin \left(2 \pi \xi_{1}\right) \sqrt{1-\eta_{1}^{2}} \\
\vec{x}_{1}=\vec{x}_{s}+R \hat{n}_{1},
\end{gathered}
$$

where $\eta_{1}$ is uniform on $[-1,1]$ and $\xi_{1}$ is uniform on $[0,1]$. This describes a procedure for choosing boundary constraints for a BSF, but the FPI approach is general to other geometry as well. Here, $s$ is a free variable, but it is useful to control its distribution. Therefore we enforce that $s$ is uniform on the interval $\left[\left\|\vec{x}_{1}-\vec{x}_{0}\right\|, s_{\max }\right]$ where $s_{\max }$ is left free.

Next, we are tasked with finding a space curve $\mathbf{r}(t)$ which satisfies the randomized boundary constraints. More formally, $\mathbf{r}(t)$ is a space curve defined on $t \in[0,1]$ having the boundary conditions:

$$
\begin{array}{rlrl}
\mathbf{r}(0) & =\vec{x}_{0} & \mathbf{r}(1) & =\vec{x}_{1} \\
\frac{\partial}{\partial t} \mathbf{r}(0) & =\hat{n}_{0} & \frac{\partial}{\partial t} \mathbf{r}(1) & =\hat{n}_{1} .
\end{array}
$$

We impose the additional constraint that there is an arc length parameterization of the space curve $\mathbf{r}^{\prime}\left(s^{\prime}\right)$ and it is defined on $s^{\prime} \in[0, s]$. To be discretized properly we must have that

$$
\vec{x}_{1}=\vec{x}_{0}+\Delta s \sum_{i=0}^{M-1} \frac{\partial}{\partial s^{\prime}} \mathbf{r}^{\prime}(i \Delta s)
$$

where $\Delta s=s / M$, with $M$ being path subdivision count. 
There are a number of different ways such a curve can be computed. We find using Bézier curves is an attractive option due to their ability to control both the position and tangent vector at the control vertices. The arc length may be varied by placing additional control vertices at different positions. We use a form for $n$-degree Bézier curves. $\mathbf{B}(t)$ [16, Chapter 10] is defined on $t \in[0,1]$,

$$
\mathbf{B}(t)=\sum_{i=0}^{n}\left(\begin{array}{c}
n \\
i
\end{array}\right)(1-t)^{n-i} t^{i} \vec{p}_{i},
$$

where $\vec{p}_{i}$ is one of $n+1$ control vertices. We can satisfy the constraints by constructing a Bézier curve with the following control vertices:

$$
\begin{gathered}
\vec{p}_{0}=\vec{x}_{0} \\
\vec{p}_{1}=\vec{x}_{0}+\ell_{0} \hat{n}_{0} \\
\vec{p}_{2}=\left(\vec{x}_{0}+\vec{x}_{1}\right) / 2+\ell_{2} \hat{n}_{2} \\
\vec{p}_{3}=\vec{x}_{1}-\ell_{1} \hat{n}_{1} \\
\vec{p}_{4}=\vec{x}_{1} .
\end{gathered}
$$

Here, $\ell_{0}$ and $\ell_{1}$ are both independent uniform random variables, each distributed over the interval $[0,0.5 R]$, and $\ell_{2}$ will be computed. This properly constrains both the position and tangent vector at the space curve's endpoints. The multiplication of the random scalars adds some measure of arc length variation. Figure 1 offers a diagram showing the relationship between the terms.

One last constraint is to ensure the space curve $\mathbf{B}$ has arc length $s$. The $\ell_{2}$ parameter can enforce this constraint. Choose $\hat{n}_{2}$ via spherical uniform sampling. Form a bracketing interval on $s$ by allowing $\ell_{2}$ to be any value in the range $\left[0, \ell_{\max }\right]$. Once this bracketing interval is established, use any root finding mechanism to find a $\ell_{2}$ such that the arc length of $\mathbf{B}$ is $s$. Brent's method is suitable for this step, and $\ell_{\max }$ can be any sufficiently large static number. Alternatively, $\ell_{\max }$ could be dynamically computed via a doubling procedure.

The FPI approach relies on a form of discretized Frenet-Serret curve which is detailed in [3]. We must convert the space curve to this discretized form to use it. The first parameter is the initial position, $\vec{x}_{0}=\mathbf{r}(0)$. Similarly, we must find the initial tangent vector:

$$
\mathbf{T}_{0}=\hat{n}_{0}=\frac{\partial}{\partial t} \mathbf{r}(0)
$$

The remainder of the orthonormal frame is the Frenet-Serret frame:

$$
\begin{gathered}
\mathbf{N}_{0}=\frac{\partial^{2}}{\partial t^{2}} \mathbf{r}(0) \\
\mathbf{B}_{0}=\mathbf{T}_{0} \times \mathbf{N}_{0}
\end{gathered}
$$

Finally, it is left to define the step size, $\Delta s$. We define this with respect to the number of desired path subdivisions, $M$, which remains a free parameter:

$$
\Delta s=s / M .
$$

We continue to find an arc length parameterization of the space curve. The arc length parameterization is necessary due to the path integral formulation requiring constant step sizes $\Delta s$. Calculating arc length parameterizations is not trivial because the arc length function is not invertible. The arc length function $a(t)$ of our space curve is defined

$$
a(t)=\int_{0}^{t} d t^{\prime}\left\|\frac{\partial}{\partial t^{\prime}} \mathbf{r}\left(t^{\prime}\right)\right\|
$$

and given our space curve is defined by Equation (10) it is not invertible analytically. We have to rely on numerical methods.

Finding arc length parameterizations of Bézier curves and parametric curves in general is an area of much interest. Many approaches seek to find approximate solutions for a speed trade-off $[17,18]$. Guenter and Parent [19] numerically calculate $a^{-1}(s)$ in a table to find bracketing intervals. The bracketing intervals aid a Newton solver in computing a more exact solution.

Our solution, which is similar to Guenter and Parent's, follows. Choose a positive integer $k$ which becomes the size of the inverse arc length table. Ideally $k$ should be a number much less than $M$, as the idea is to reduce the number of inverse arc length calculations from $M$ down to 


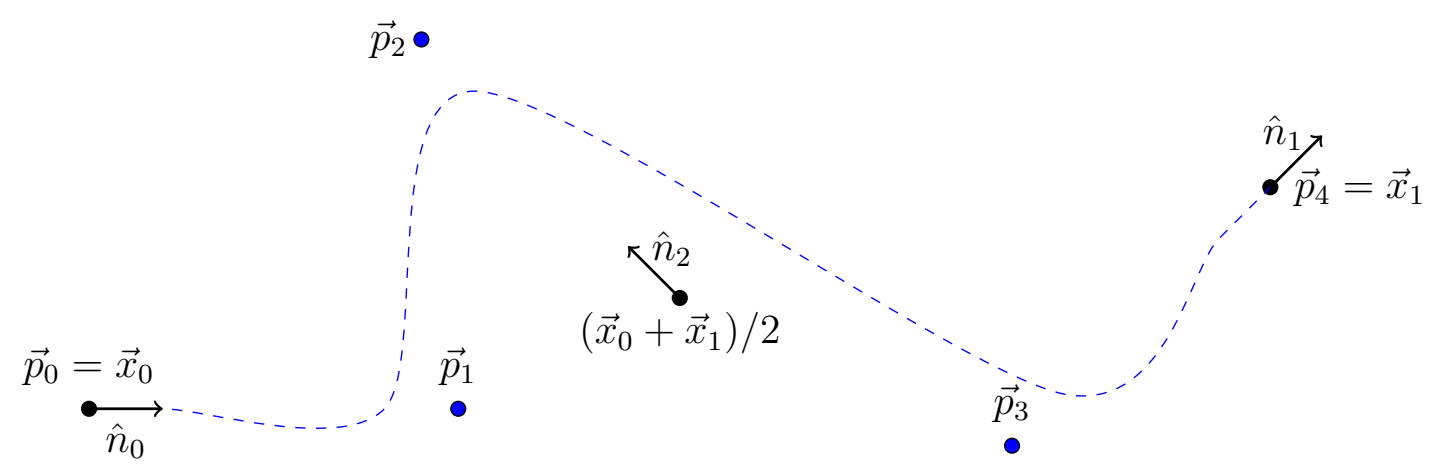

Figure 1: Diagram of Bézier curve control points. Here, $\vec{x}_{0}$ and $\vec{x}_{1}$ are chosen directly from the light and sensor geometry and serve as control points. We compute $\vec{p}_{1}$ and $\vec{p}_{3}$ as a function of these two points. $\vec{p}_{2}$ is chosen randomly by starting at the midpoint of $\vec{x}_{0}$ and $\vec{x}_{1}$ and tracing a distance $\ell_{2}$ out from a spherically uniform vector $\hat{n}_{2}$.

$k$. For example, we use $k=M / 20$. Choose a sequence of arc lengths to calculate $a_{j}=j \times s / k$. Form a table of the matching parameter-space values by solving the inverse arc length function numerically with Brent's method:

$$
\begin{aligned}
& t_{1}=a^{-1}\left(a_{1}\right) \\
& t_{2}=a^{-1}\left(a_{2}\right) \\
& \cdots \\
& t_{k-1}=a^{-1}\left(a_{k-1}\right) \\
& t_{k}=a^{-1}\left(a_{k}\right) .
\end{aligned}
$$

Brent's method can solve the inverse arc length function by optimizing $a(t)-s=0$ for $t$. Once the table is calculated, form a second sequence of arc lengths $s_{i}=i \times s / M$. For each $s_{i}$ there exists a $a_{j}$ and a $a_{j+1}$ such that $a_{j}<s_{i} \leq a_{j+1}$. The corresponding curve parameter can be approximated with linear interpolation. Let $q=$ $\left(s_{i}-a_{j}\right) /\left(a_{j+1}-a_{j}\right)$ :

$$
t_{i}=q t_{j}+(1-q) t_{j+1} .
$$

At this point, our technique differs from Guenter and Parent. They use the interpolated value to serve as an estimate for a root solver, and find a precise value by root finding over the subinterval. In practice, root-finding on this subinterval is quite slow for our purposes. Instead, we correct for the error after the curve is discretized. We sample the curvature function $\kappa(t)$ and the torsion function $\tau(t)$ along the sequence $t_{i}$. This generates the curve parameters

$$
\kappa_{i}=\kappa\left(t_{i}\right) \quad \tau_{i}=\tau\left(t_{i}\right) .
$$

These form the last remaining discrete FrenetSerret parameters.

The remaining portion of the numerical methods is to repeatedly perturb these initial curves using an existing perturbation algorithm. The general idea is to choose three path segments $i<j<k$ at random, modify $\kappa_{j}$ randomly, and allow a root solver to change the remaining curvature and torsion values such that the boundary constraints are preserved. The algorithm is detailed in [9] and [3].

The transport kernel can be evaluated via Monte Carlo integration. In [9] an expression is given for a single segment of the discrete FrenetSerret curve, written $\omega_{j}$ for the $j$-th segment, which is derived from Equation (6). We can generate a sequence of paths using the aforementioned perturbation method and write the path segment weight as $\omega_{i, j}$ for the $j$-th segment of the $i$-th path. A path's weight is the product of its segment weights. Monte Carlo integration of the transport kernel is then

$$
G\left(s, \vec{x}_{1}, \hat{n}_{1}, \vec{x}_{0}, \hat{n}_{0}\right)=\lim _{N \rightarrow \infty} \frac{1}{N} \sum_{i=1}^{N} \prod_{j=1}^{M} \omega_{i, j} .
$$

To calculate a beam spread function, each one of these path integral calculations can be mapped to bins over an angle range in $\theta$. Each bin then includes path integral calculations corresponding 


\begin{tabular}{cc}
\hline Parameter & Value \\
\hline$a$ & $0.004 \mathrm{~cm}^{-1}$ \\
$b$ & $0.1 \mathrm{~cm}^{-1}$ \\
$R$ & $30 \mathrm{~cm}$ \\
$s_{\max }$ & $100 \mathrm{~cm}$ \\
$\mu$ & 0.5 \\
$\epsilon$ & 0.075 \\
\hline
\end{tabular}

Table 1: Table of experiment parameters used to reconstruct Maffione BSF measurements.

to that $\theta$ over a range of $s$. We can then perform a similar Monte Carlo integration procedure to integrate over $s$ for that bin.

\section{Results}

A BSF is a straightforward scenario to test a new fundamental technique. A collimated light source, such as a laser, is suspended in a scattering media aimed at a radiometer, placed a distance $R$ apart. The light source is then rotated a positive and negative angle amount, $\theta$, and the irradiance is measured with respect to $\theta$.

Experimentally measured BSFs include, for instance, ocean water and sea ice. We compare our calculated BSF against a measured sea ice BSF [11]. Because many parameters are not known, they are estimated for the purpose of replication. The scattering coefficient $(b)$ and absorption coefficient $(a)$ are chosen based on the relatively scattering nature of sea ice as compared to its absorption properties. The phase function is not known, so we assume a forward-peaked Gaussian parameterized by $\mu$ and $\epsilon$. Here, $\mu$ is the width of the Gaussian and $\epsilon$ is a numerical parameter detailed in [3] which aids in the tractability of the weight calculation. In the limit of $\epsilon \rightarrow 0$, the calculation approaches theory. We also consider a maximum arc length $s_{\max }$ which is ten scattering lengths. A table of parameters is given in Table 1 .

Using these experimental parameters, we calculate a BSF in an attempt to compare to experimental data (Figure 2). There are shared characteristics between the calculated and experimental BSFs: the peak at $0^{\circ}$ and gradual decrease in the relative intensity in the mid-range angles. The gradual falloff suggests some of the multiple scattering behavior is being captured. The calculated BSF falls off to about 0 by the $90^{\circ}$ mark, whereas experimental results still measure some amount of intensity at the extremes.

To compare more formally, we attempt to fit models used by Maffione et al. to the calculated BSF. They report using a Lorentzian fit for their measurements, but also use a Gaussian as a first effort. For the calculated BSF, a Gaussian fits fairly well (Figure 3) whereas the Lorentzian function did not. This suggests a shape difference in the calculated and experimental BSFs. A key difference between the calculated and experimental results is the broadness. A possible metric for this characteristic is the width parameter $\sigma$ for the Gaussian fit. We calculated BSFs for varying path segments $M$ with the hypothesis that this simulation parameter affects the fit parameter $\sigma$. The reason for choosing $M$ is its inverse relationship with $\Delta s$. Smaller values of $\Delta s$ approximate the path integral more exactly. We choose smaller values $(M=\{80,120,160,200\})$ due to present floating point precision difficulties extending the technique to higher values of $M$.

The results of this experiment are presented in Table 2. There is an increase in the width of the Gaussian as more path segments are used. The fit width $\sigma$ appears to approach a value in the limit of $M$. Step functions offer a way to project this value. The step function

$$
\sigma=A\left(M^{p} /\left(B+M^{p}\right)\right)
$$

is a possible fit for $p=4$ on our limited data set. See Figure $4 \mathrm{~b}$ for the resulting fit. Projecting the value of $\sigma$ in the limit of $M$ yielded a value of $\sigma=$ 23.78. However, plotting such a Gaussian against the $M=200$ data set (Figure 4c) shows little difference. Drawing conclusions from such a small data set is difficult, but we suspect computing BSFs for higher $M$ will have diminishing returns.

\section{Conclusion}

We introduce an algorithm for constructing initial paths for use in the FPI approach to calcu- 


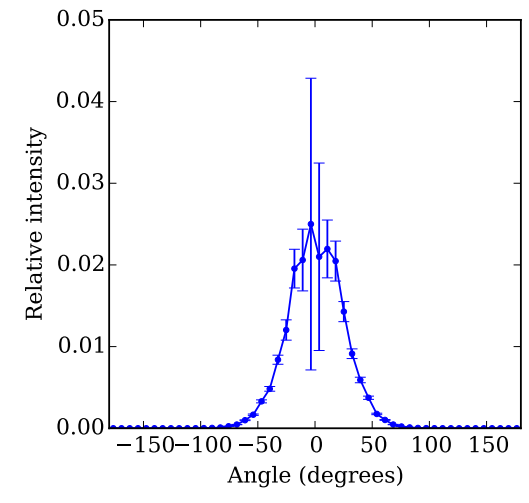

(a) Calculated BSF for $M=200$.

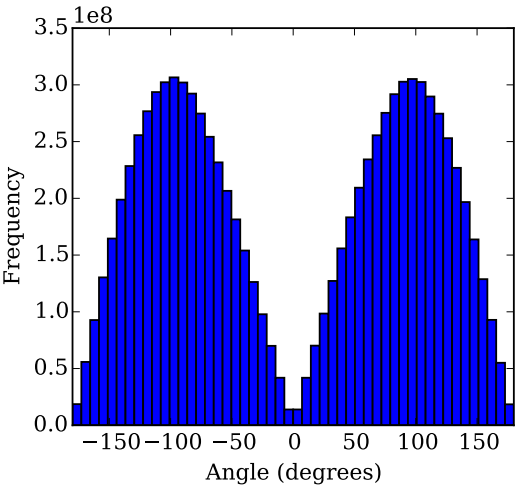

(b) Sample pattern for (a).

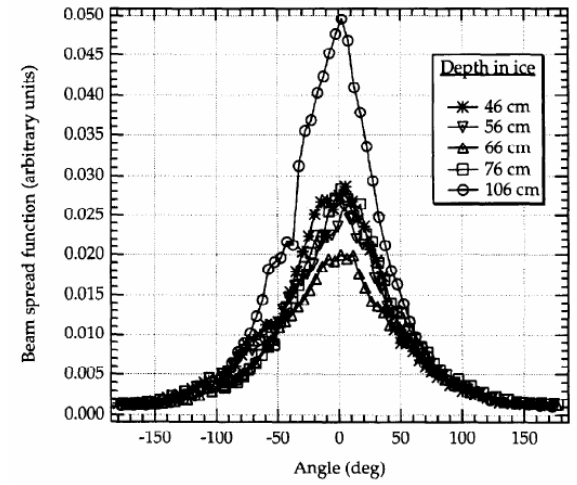

(c) Experimental BSFs (Figure 5 from [11].)

Figure 2: A calculated BSF (a) using the numerical FPI approach, normalized such the peak value is 0.025 . The sample pattern shown in (b) is the reason for the increased standard error of the mean in (a). In (c) we see similar trends as in (a) but especially at the $50^{\circ}$ mark there is disagreement in the shape. The calculated BSF does not have the same gradual falloff of the experimental BSFs.

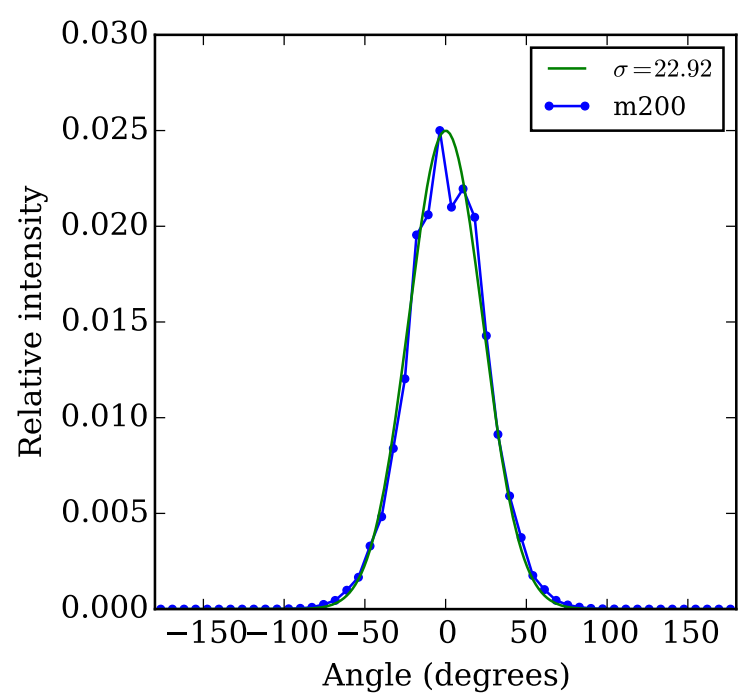

(a) Gaussian fit for $M=200$ data set.

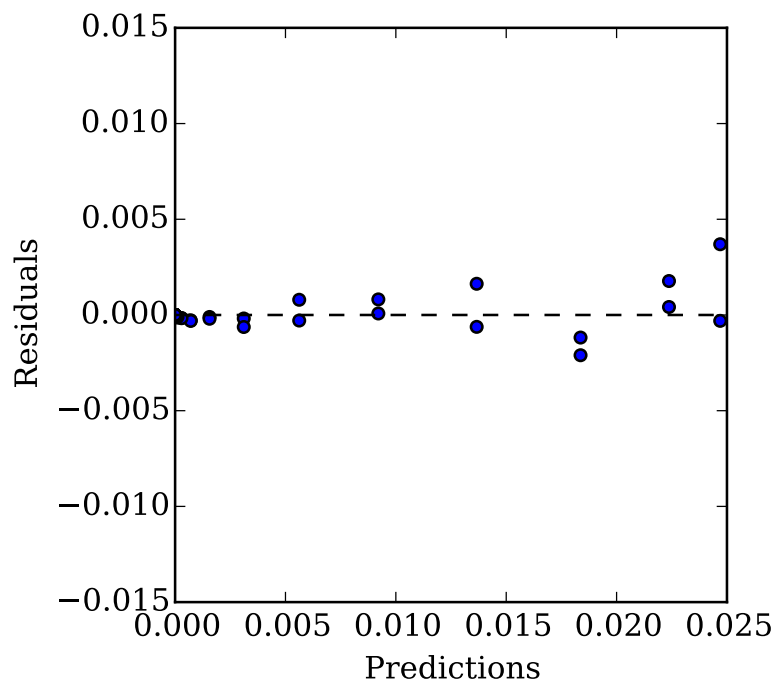

(b) Residuals plot.

Figure 3: Fit and residuals for $M=200$ data set. Visually, the fit appears decent. The residuals break down for higher-value predictions which in this case correspond to smaller angles. It is possible the model breaks down again due to undersampling in the small angles. 


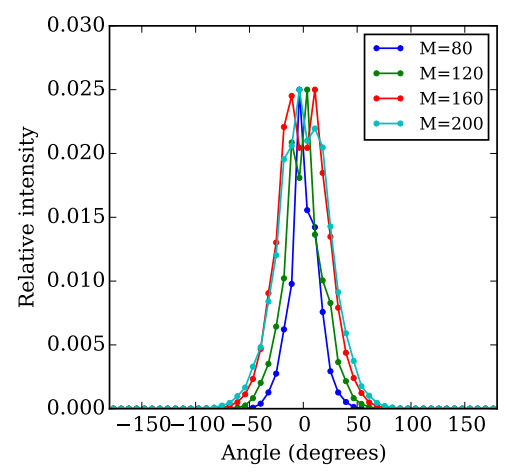

(a) All calculated BSFs.

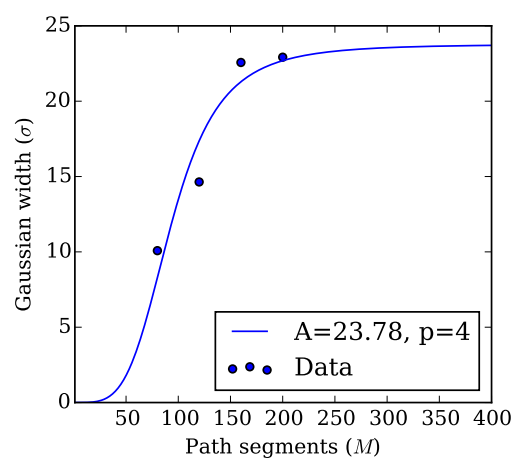

(b) Gaussian width projection.

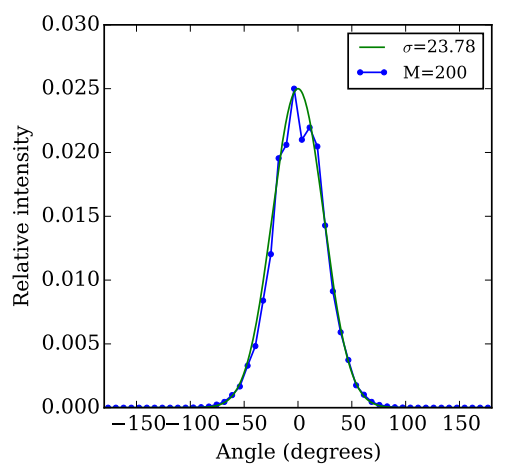

(c) $M=200$ data set with $M=\infty$ Gaussian.

Figure 4: Calculated BSFs for varying path segments $M$ (a) and the projection of the Gaussian fit width $\sigma$ (b). Assuming the step function is correct, the Gaussian fit width would approach 23.78 in the limit of $M$. Comparing this projection with the $M=200$ data set (c) shows little difference. This can mean the step function is inappropriate, there are not enough data to obtain a good fit, or calculating a BSF for higher $M$ would yield little change.

\begin{tabular}{cc}
\hline Path segments $(M)$ & Gaussian width $(\sigma)$ \\
\hline 80 & 10.1 \\
120 & 14.6 \\
160 & 22.6 \\
200 & 22.9 \\
\hline
\end{tabular}

Table 2: Result of the path segment variation experiment. The parameters given in Table 1 are used again in this experiment. Inspection suggests $M$ does have a strong effect on $\sigma$.

lating radiative transfer. The new method is used to calculate numerically beam spread functions. This represents the most complex experiment conducted to date with the FPI approach. The calculations are compared to measured BSFs of sea ice and are found to be narrower. Lorentzian fits for the calculated BSFs were found inadequate whereas a Gaussian function fits the calculated BSFs nicely, a departure from the measured BSFs of Maffione et al. We also see disagreement in the large angles, where our calculated BSFs report intensity close to zero and the measured BSFs show some intensity in the large angles. This may be because the phase function we use is a forwardpeaked Gaussian which does not support much backscatter, and this may decrease intensity in the larger angles. The width of the Gaussian appears to approach a value as the path segment count increases. A projection for this value is attempted and suggests diminishing returns for calculating the BSF for greater numbers of path segments.

Future work using the FPI approach to calculate BSFs could compare against other BSF data, or continue the trend of calculating BSFs with increasing numbers of path segments. However, a single BSF takes the FPI approach about 40 compute-years to calculate and keep variance under control. This might be remedied by flattening the sample distribution with respect to the BSF angle (see Figure 2b) or to bring the solution to other parallel architectures like GPGPU. Currently the FPI approach is only limited by the amount of compute time available, as it is an embarrassingly parallel problem. Other parameters could be the subject of sensitivity studies, such as number of path perturbations, the maximum arc length $\left(s_{\max }\right)$, and total path count. Improvements and approximation to the numerical techniques introduced here could be studied. For example, the arc length parameterization takes a significant portion of time to calculate but is not always accurate - it could be omitted and the error corrected for by the perturbation step. Standard acceleration techniques for Monte Carlo approaches apply to the numerical FPI approach, such as importance sampling and the Metropolis 
algorithm. We expect their adoption will be an important development.

The results in this article show numerical plausibility in the new extensions to the FPI approach. They show that the FPI approach is ready to be tested with more realistic phase functions, against known benchmarks, and more experimental data sets. We continue to know little about the FPI approach's physical accuracy until this future work is completed, but the Monte Carlo method shows promise.

\section{Acknowledgments}

This research employed resources provided by the Open Science Grid [20], which is supported by the National Science Foundation and the U.S. Department of Energy's Office of Science.

The authors gratefully acknowledge the efforts of the Clemson University Cyberinfrastructure Technology Integration (CITI) team for providing early computing resources for this work.

\section{References}

[1] J. Tessendorf, Radiative transfer as a sum over paths, Physical Review A 35 (2) (1987) 872. doi:10.1103/ PhysRevA.35.872.

[2] J. Tessendorf, Time-dependent radiative transfer and pulse evolution, JOSA A 6 (2) (1989) 280-297. doi: 10.1364/JOSAA.6.000280.

[3] J. Tessendorf, Numerical integration of the Feynman path integral for radiative transport, in: International Conference on Mathematics, Computational Methods and Reactor Physics, Saratoga Springs, NY, 2009.

[4] J. Tessendorf, Angular smoothing and spatial diffusion from the Feynman path integral representation of radiative transfer, Journal of Quantitative Spectroscopy and Radiative Transfer 112 (4) (2011) 751 760. doi:10.1016/j.jqsrt.2010.11.004.

[5] L. T. Perelman, J. Wu, I. Itzkan, M. S. Feld, Photon migration in turbid media using path integrals, Physical Review Letters 72 (9) (1994) 1341. doi: 10.1103/PhysRevLett.72.1341.

[6] L. T. Perelman, J. Wu, Y. Wang, I. Itzkan, R. R. Dasari, M. S. Feld, Time-dependent photon migration using path integrals, Physical Review E 51 (6) (1995) 6134. doi:10.1103/PhysRevE.51.6134.

[7] L. T. Perelman, J. Winn, J. Wu, R. R. Dasari, M. S. Feld, Photon migration of near-diffusive photons in turbid media: a Lagrangian-based approach, JOSA A 14 (1) (1997) 224-229. doi:10.1364/JOSAA. 14. 000224 .
[8] J. N. Winn, L. T. Perelman, K. Chen, J. Wu, R. R. Dasari, M. S. Feld, Distribution of the paths of earlyarriving photons traversing a turbid medium, Applied optics 37 (34) (1998) 8085-8091. doi:10.1364/A0. 37.008085.

[9] P. Kilgo, J. Tessendorf, Accelerated path generation and visualization for numerical integration of Feynman path integrals for radiative transfer, in: Joint International Conference on Mathematics and Computation, Supercomputing in Nuclear Applications and the Monte Carlo Method, 2015.

[10] H. R. Gordon, Equivalence of the point and beam spread functions of scattering media: a formal demonstration, Applied optics 33 (6) (1994) 1120-1122. doi: 10.1364/A0.33.001120.

[11] R. A. Maffione, J. M. Voss, C. D. Mobley, Theory and measurements of the complete beam spread function of sea ice, Limnology and Oceanography 43 (1) (1998) 34-43. doi:10.4319/1o.1998.43.1.0034.

[12] S. Q. Duntley, Underwater lighting by submerged lasers and incandescent sources, Tech. rep., Scripps Institution of Oceanography (Jun. 1971).

[13] L. Mertens, F. Replogle Jr, Use of point spread and beam spread functions for analysis of imaging systems in water, JOSA 67 (8) (1977) 1105-1117. doi:10. 1364/JOSA . 67.001105.

[14] K. J. Voss, Variation of the point spread function in the Sargasso Sea, in: San Diego,'91, San Diego, CA, International Society for Optics and Photonics, 1991, pp. 97-103. doi:10.1117/12.48874.

[15] J. Schoonmaker, K. Voss, G. Gilbert, Laboratory measurements of optical beams in young sea ice, Limnology and oceanography 34 (8) (1989) 1606-1613. doi:10.4319/1o.1989.34.8.1606.

[16] R. H. Bartels, J. C. Beatty, B. A. Barsky, An Introduction to Splines For Use In Computer Graphics and Geometric Modeling, 1st Edition, Morgan Kaufmann Publishers, Inc., 2929 Campus Drive, San Mateo, CA 94403, 1987.

[17] M. Madi, Closed-form expressions for the approximation of arclength parameterization for Bezier curves, International Journal of Applied Mathematics and Computer Science 14 (1) (2004) 33-42.

[18] H. Wang, J. Kearney, K. Atkinson, Arc-length parameterized spline curves for real-time simulation, in: 5 th International Conference on Curves and Surfaces, 2002 .

[19] B. Guenter, R. Parent, Computing the arc length of parametric curves, IEEE Computer Graphics and Applications 10 (3) (1990) 72-78. doi:10.1109/38. 55155.

[20] R. Pordes, D. Petravick, B. Kramer, D. Olson, M. Livny, A. Roy, P. Avery, K. Blackburn, T. Wenaus, F. Würthwein, et al., The Open Science Grid, in: Journal of Physics: Conference Series, Vol. 78, IOP Publishing, 2007, p. 012057. 